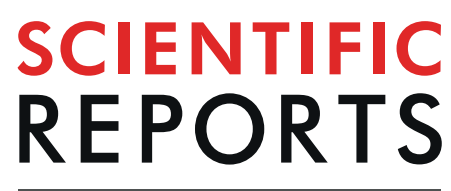

natureresearch

Check for updates

\title{
Free space super focusing using all dielectric hyperbolic metamaterial
}

\begin{abstract}
Norhan A. Salama ${ }^{1,2,3}$, Mai Desouky², S. S. A. Obayya ${ }^{3}$ \& Mohamed A. Swillam $^{2 \bowtie}$
Despite that Hyperbolic Metamaterial (HMM) has demonstrated sub-wavelength focusing inside of it, sub-wavelength imaging in free space of HMM is rarely introduced. The decay of hyperbolic momentum space outside the hyperbolic medium has hindered the realization of sub-wavelengh focusing in the near field of HMM. Furthermore, manipulating the negatively refracted waves exiting the HMM have addressed another major obstacle to realize free space sub-wavelength focusing. In this work, we report extended sub-wavelength focusing in free space based on negative refraction of light exiting the HMM. The proposed structure is composed of multilayers of doped InAs/intrinsic InAs integrated with metallic slit. We theoretically simulate the doped InAs/intrinsic InAs HMM and investigate the negative refraction behavior outside the HMM. We optimized the structure for achieving high resolution down to $0.2 \lambda$, extended to a distance of $3.2 \mu \mathrm{m}$ in free space. Also, sub-wavelength focusing in free space has been studied at different doping concentrations showing that the small doping concentrations exhibit enhancement in resolution at short distances up to $600 \mathrm{~nm}$ away from the HMM. Extending the focusing distance is achieved up to distance $3.5 \mu \mathrm{m}$ from the hyperbolic structure by manipulating the doping concentration. This proposed lens configuration is expected to find potential usage in mid IR thermal imaging and photolithography application.
\end{abstract}

Imaging with conventional optical systems is constrained with the diffraction limit, where the maximum resolution can be reached is at most half of the incident wavelength. Imaging is based on retrieving the scattering amplitudes and phase shifts of various momentum modes. In ordinary isotropic medium, high orders of momentum components (high $k$-modes) -which are larger than those of free space $\left(\mathrm{K}_{\mathrm{o}}\right)$-exponentially decay in the vicinity of the object, leading to exponential small light interaction with the object. Consequently, the scattering from these modes are negligible and evanescent in free space, thus, they can't contribute to the formation of the final image of an object. These high $k$-modes carry sub-wavelength features of the object ${ }^{1}$. In order to beat the diffraction limit, it is required to recover the high order momentum modes that are exponentially decayed in free space, thus, retrieving the sub-wavelength features of an object. Many studies have introduced different approaches to overcome the diffraction limit such as the contact mask and scanning near field optical microscopy (SNOM) ${ }^{2,3}$. However, they all suffer from the drawbacks of low throughput and long post processing. Alternatively, metamaterial, as first proposed by Veselago $(1968)^{4}$ represents a new paradigm striking the electromagnetic features in which it exhibits negative refraction of light, a property that cannot be attained before. The super lens, proposed by sir.Pendry ${ }^{5}$, was the first demonstration of Veslago's theory enabling sub-diffraction resolution imaging in microwave wavelength range. Although, super lens is a successful approach overcoming the diffraction limit, serious constrains are imposed by nanofabrication techniques, when uplifting the working frequencies of super lens up to optical ranges ${ }^{6}$. That is in addition to the difficulty to obtain negative magnetic permeability in the visible domain ${ }^{7}$. Inspired by Pendrys' work, different studies have developed different approaches for tailoring the electric permittivity and magnetic permeability and hence the index of refraction. These approaches find multiple functionalities such as invisibility cloacking ${ }^{8}$, perfect absorption ${ }^{9}$, digital coding ${ }^{10-12}$, negative refraction $^{13}$ sub-diffraction focusing ${ }^{14-18}$. Hyperbolic metamaterials (HMMs) can act as an alternative solution to achieve sub-diffraction focusing without the need to tune the magnetic permeability or implementing complex designs. HMM is a composite structure that displays a dispersion relation where the material behaves as a metal in one direction in space and as a dielectric in the other direction ${ }^{19,20}$, such material is also called indefinite media. Hyperlens, a curved structure that can be used for sub-diffraction imaging in the far field ${ }^{21}$. However, the difficulty of fabricating the curved hyperlens restricts its practical application. Despite of the demonstration of numerous studies concerning sub-diffraction focusing inside the HMM medium ${ }^{22-26}$, lensing in the near field

${ }^{1}$ Laser Application in Metrology, Photochemistry \& Agriculture, National Institute of Laser Enhanced Sciences, Cairo University, Giza, Egypt. ${ }^{2}$ Department of Physics, The American University in Cairo, Cairo, 11835, Egypt. ${ }^{3}$ Centre for Photonics and Smart Materials, Zewail City of Science and Technology, Giza, Egypt. ${ }^{\bowtie}$ e-mail: m.swillam@aucegypt.edu 
of HMMs is rarely introduced in real structures except for few attempts - such as Si pillars arrays in the THz range $^{27}$ - providing minor focusing resolution. The challenge of near field focusing outside the HMM is imposed by the rapid decay of the evanescent waves in free space. Nonetheless, HMM is characterized by extremely large optical density of states that extended to the near field of HMM and consequently do contribute to the propagation of large wave vectors outside the HMM that is in turn, prevents the rapid decay of the evanescent field ${ }^{28}$. This phenomenon is accompanied with subsequent negative refraction of the electromagnetic (EM) waves exiting the HMM, which are the key roles for achieving sub diffraction focusing in the near field of $\mathrm{HMM}^{6}$. In this work, we report near field focusing in free space with sub-diffraction focusing resolution based on a single planar HMM lens composed of doped InAs/intrinsic InAs integrated with upper metallic slit. The proposed design can be fabricated using conventional 2D-fabrication technologies such as e-beam lithography and evaporation of metal films, or using direct Laser writing ${ }^{29-31}$, the procedure has been explicitly described in previous reference ${ }^{32}$.

We demonstrate the sub-diffraction focusing effect based on paraxial negative refraction (small angle of refraction) across the second interface of the HMM lens for structure of doping concentration $\left(\mathrm{N}_{\mathrm{d}}\right) 7.5 \times 10^{19} \mathrm{~cm}^{-3}$. Sub-diffraction focusing of resolution down to $0.16 \lambda$ at $\lambda=7.6 \mu \mathrm{m}$ has been achieved at focusing plane lies $500 \mathrm{~nm}$ away from the HMM lens. In addition, we report tuning the focusing distance away from the HMM lens through optimizing the slit parameters with maximum distance reached is $3.2 \mu \mathrm{m}$ at wavelength of $7.6 \mu \mathrm{m}$ and resolution down to $0.2 \lambda$. Furthermore, we investigate the focusing effect of different doping concentrations showing that the maximum extended projection for focusing is $3.5 \mu \mathrm{m}$ achieved for $\mathrm{N}_{\mathrm{d}}$ of $7.5 \times 10^{19} \mathrm{~cm}^{-3}$ at $17.74 \mu \mathrm{m}$.

\section{Theoretical Design}

The structure is composed of 10 alternating layers of doped/intrinsic InAs layers of $20 \mathrm{~nm}$ each, integrated with upper metallic slit (copper slit), see Fig. 1a. In order to excite the high wave vectors in a HMM, a diffraction grating or Fresnel zone plate is applied on top of the structure. The upper slit $\boldsymbol{w}$ is tuned for optimum focusing. We define the permittivity tensor components as diagonal tensor with $\left(\varepsilon_{\mathrm{xx}}, \varepsilon_{\mathrm{yy}} \varepsilon_{\mathrm{zz}}\right)$ corresponds to $\left(\varepsilon_{/ /,}, \varepsilon_{/ /}, \varepsilon_{\perp}\right)$. The parallel permittivity $\left(\varepsilon_{/ /}\right)$corresponds to the in-plane component and the perpendicular permittivity $\left(\varepsilon_{\perp}\right)$ corresponds to the out of plane component. The commercial software finite difference time domain-Lumerical-is used for theoretical investigation ${ }^{33}$. TM polarized Gaussian beam of NA $=0.2$ is incident from the top of the structure providing paraxial incidence (small angle of incidence). Perfect matched layers (PML) are used as the boundaries in the $\mathrm{x}$ and $\mathrm{z}$ directions. The structure has sub-wavelength dimensions, so, its effective parameters could be extracted by homogenization using the effective medium approximation (EMA) theory as follows ${ }^{34}$ :

$$
\begin{aligned}
& \varepsilon_{\perp}=\frac{\varepsilon_{\text {doped }} \cdot \varepsilon_{d}}{f_{1} \varepsilon_{\text {doped }}+f_{2} \varepsilon_{d}} \\
& \varepsilon_{\|}=f_{1} \varepsilon_{\text {doped }}+f_{2} \varepsilon_{d}
\end{aligned}
$$

where $\varepsilon_{\text {doped }}$ and $\varepsilon_{d}$ are the permittivities of doped and intrinsic InAs respectively. The filling ratios $f_{1}$ and $f_{2}$ are chosen to be 0.5 . The permittivity of intrinsic InAs is $\varepsilon_{\mathrm{d}}=3.937$, while the permittivity of doped InAs is calculated using the Drude modal.

$$
\varepsilon_{\text {doped }}=\varepsilon_{\infty}-\frac{\omega_{p}^{2}}{\omega^{2}+i \omega^{2} \Gamma}
$$

where $\varepsilon_{\infty}=15.5, \omega_{p}$ is the plasma frequency and $\Gamma$ is the scattering rate. The values of $\omega_{p}$ and $\Gamma$ are extracted from previous literatures ${ }^{27,35}$. The dispersion relation of the structure comprising InAs of doping concentration $\mathrm{N}_{\mathrm{d}}$ $7.5 \times 10^{19} \mathrm{~cm}^{-3}$ is presented in Fig. 1b. The hyperbolic region of type (II) where $\left(\varepsilon_{/ /}<0\right.$ and $\left.\varepsilon_{\perp}>0\right)$ is the region of interest throughout our whole study (marked by blue circle in Fig. 1b).

The key for near field sub-diffraction focusing using HMM is based on two aspects: the first aspect is, the extreme anisotropic nature of HMM, i.e. where $\varepsilon_{z z} \cdot \varepsilon_{x x}<0$, that enables the sustained propagation of high $k$-modes inside the HMM and could be extended to a distance in the near field (outside the HMM) comprising the high optical density of states. The extreme anisotropic nature of HMM gives rise to open hyperboloid iso-frequency contour, as demonstrated in Fig. 1c, according to the dispersion relation:

$$
\frac{k_{x}^{2}+k_{y}^{2}}{\varepsilon_{z z}}+\frac{k_{z}^{2}}{\varepsilon_{x x}}=\frac{\omega^{2}}{c^{2}}
$$

The second aspect is based on the negative refraction behaviour across the interface of HMM/free space ${ }^{6,36,37}$. For paraxial incidence (verified by Gaussian beam), the excited high $k$-modes preferentially propagates inside HMM with an angle of refraction, with respect to the optical axis, defined by the ratio of components of Poynting vectors, according to the following relation ${ }^{38}$ :

$$
\theta=\arctan \frac{S_{x}}{S_{z}}=\arctan \left(\frac{\varepsilon_{x x} k_{x}}{\varepsilon_{z z} k z}\right)
$$

when $k_{x} \rightarrow \infty$, the limit of the angle is approximated as follows: 

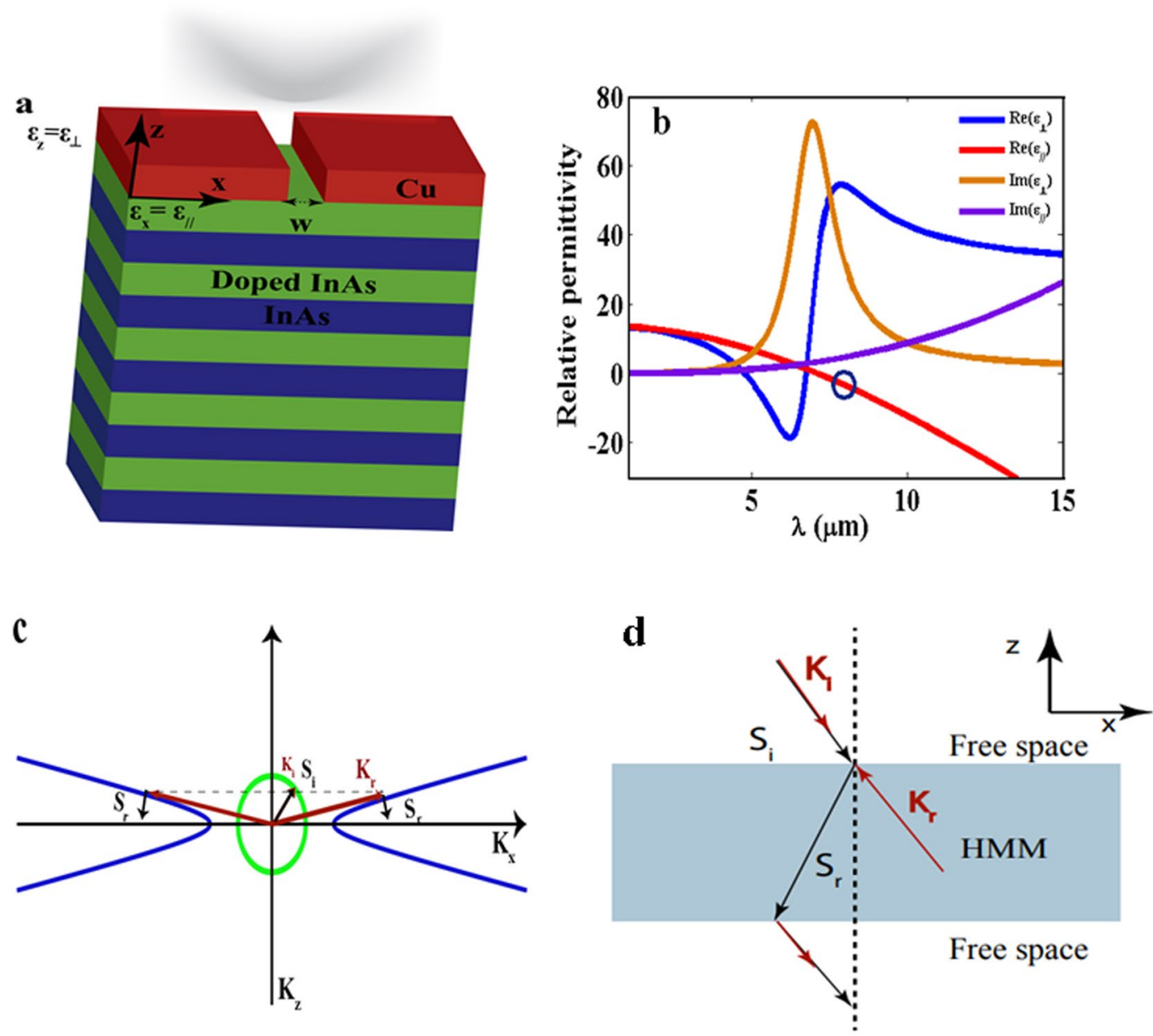

Figure 1. A schematic of the proposed HMM. It consists of 10 alternating layers of doped InAs/intrinsic InAs, with layer thickness of $20 \mathrm{~nm}$ each. A Cu layer integrated with a slit on top of the structure. The effective permittivities were defined such that $\varepsilon_{/ /}$is along the $\mathrm{x}$-axis and $\varepsilon_{\perp}$ is along the $\mathrm{z}$-axis. (b) The dispersion relation of doped InAs/intrinsic InAs of $\mathrm{N}_{\mathrm{d}}$ of $7.5 \times 10^{19} \mathrm{~cm}^{-3}$, the blue circle indicates the wavelength region of interest verifying type (П) HMM, where the real part of in-plane effective permittivity is $\varepsilon_{/ /}<0$ (red curve), while the real part of out-of-plane effective permittivity is $\varepsilon_{\perp}>0$ (blue curve). (c) The open hyperboloid equi-frequency contour at $\lambda=7.6 \mu \mathrm{m}$ with $\varepsilon_{/ /}=-\mathbf{1 . 8 8 9}$ and $\varepsilon_{\perp}=52.65$ with corresponding Poynting vector which is orthogonal to the iso-frequency contour as well as the equi-frequency contour of an isotorpic material (green circle) and (d) A schematic represents the refraction behaviour throughout the HMM lens based on the direction of Poynting vectors $(S)$ and wave vector $(K)$. The incident wave vector is given by $K_{i}$ and the incident Poynting vector is given by $\mathrm{S}_{\mathrm{i}}$. The first refraction takes place inside the HMM when the Poynting vector $\left(\mathrm{S}_{\mathrm{r}}\right)$ is negatively refracted with respect to $\mathrm{x}$-axis while the wave vector will be propagating backward. After exiting the $\mathrm{HMM}$, the $\mathrm{S}_{\mathrm{r}}$ will undergo secondary negative refraction leads to beam focusing while being parallel with the $\mathrm{K}_{\mathrm{r}}$.

$$
\theta_{\lim }=\arctan \left(\sqrt{\frac{\varepsilon_{x x}}{\varepsilon_{z z}}}\right)
$$

From Eq. (6), we can conclude that the refraction across the first HMM lens interface is a function of uniaxial parameters of the lens irrespective to the incident angle ${ }^{22}$. The first refracted modes $\left(\mathrm{K}_{\mathrm{r}}\right)$ are propagating inside the HMM lens striking the second interface of the lens and then undergoing further refraction, Fig. 1d. The negative refraction behavior can be explained upon understanding the direction of Poynting vector components $S_{x}$ (the tangential component of Poynting vector) and $\mathrm{S}_{\mathrm{z}}$ (the component in the direction of propagation representing the energy flow) across the interface. The energy flow has to be away from the interface, thus, $S_{z}>0^{39}$. The real part (Re) of the two components of Poynting vectors are represented by the following inequality equations ${ }^{38,40,41}$ :

$$
\langle S\rangle=1 / 2 \operatorname{Re}\left\langle S_{x}, 0, S_{z}\right\rangle
$$

where the $\hat{S}$ in the direction of energy flow is given by, 

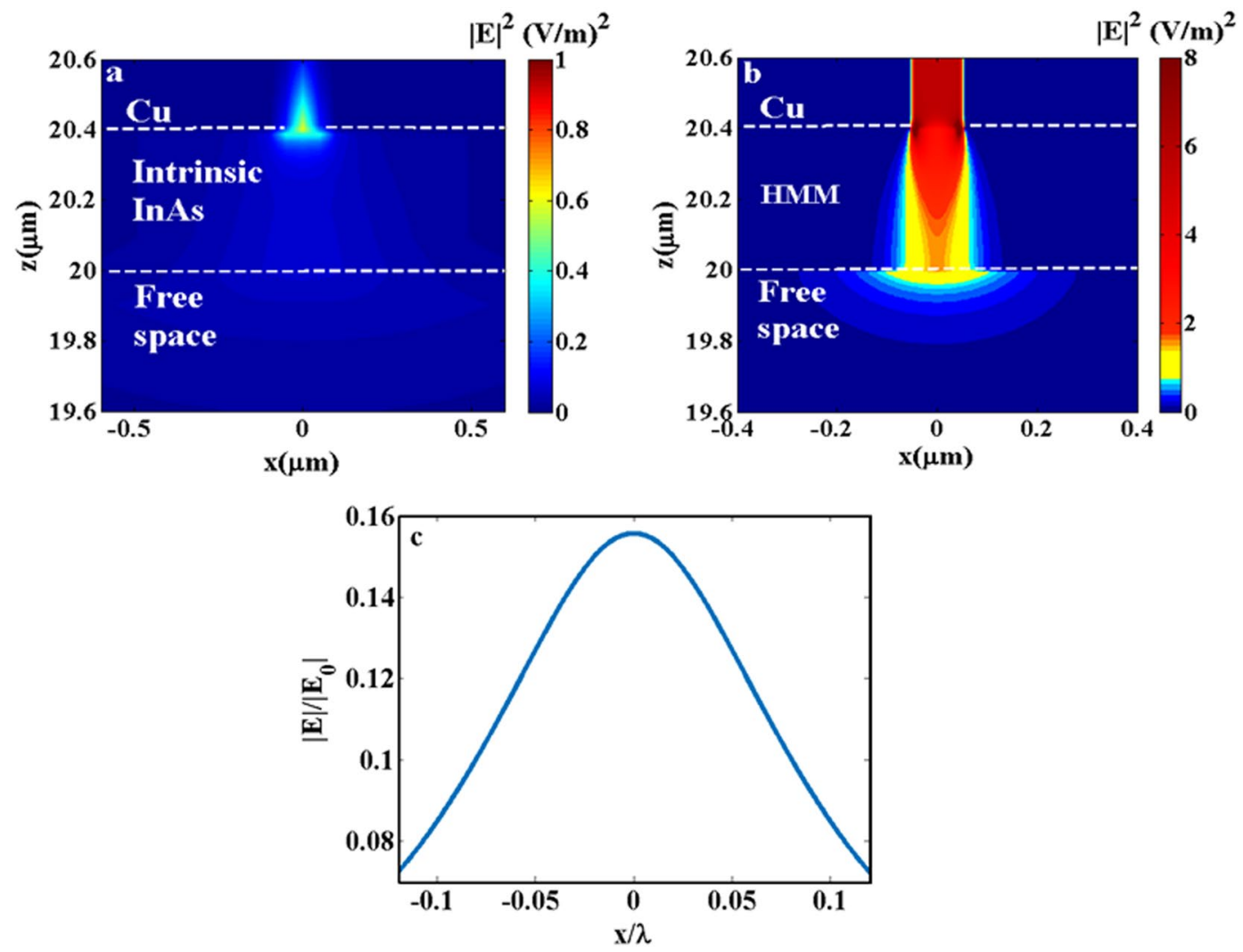

Figure 2. The electric field intensity distributions in xz-plane for: (a) Intrinsic InAs and (b) effective HMM structure, both are at $\lambda=7.09 \mu \mathrm{m}$ with $\mathrm{Cu}$ slit on top of both structures. The field can't propagate inside the intrinsic InAs while it propagates and get focused inside and outside the HMM. (c) The electric field intensity profile at a focal plane $500 \mathrm{~nm}$ away from the HMM, indicating sub-diffraction focusing of the transmitted field in free space.

$$
S_{z}=\frac{k_{z}}{\varepsilon_{x x}} \frac{H_{o}{ }^{2}}{2 \omega \varepsilon_{o}} \succ 0
$$

and the tangential component of $\hat{S}$,

$$
\begin{gathered}
S_{x}=\frac{k_{x}}{\varepsilon_{z z}} \frac{H_{o}{ }^{2}}{2 \omega \varepsilon_{o}} \prec 0 \\
\langle S\rangle=\frac{H_{o}{ }^{2}}{2 \omega \varepsilon_{o}} \operatorname{Re}\left\langle\frac{k_{x}}{\varepsilon_{z z}}, 0, \frac{k_{z}}{\varepsilon_{x x}}\right\rangle
\end{gathered}
$$

From (10), Eq. (5) can be rewritten as follows:

$$
\tan \theta=\frac{S_{x}}{S_{z}}=\left(\frac{k_{x} / \varepsilon_{z z}}{k_{z} / \varepsilon_{x x}}\right)
$$

In our study, we use a HMM where $\left(\varepsilon_{\mathrm{xx}}<0\right.$ and $\left.\varepsilon_{\mathrm{zz}}>0\right)$, thus from Eq. (11), $S_{z}$ has to be positive and the sign of $S_{x}$ flips across the interface and the energy is negatively refracted between the HMM and free space. The wave vector components become $k_{\mathrm{x}}<0$ and $k_{\mathrm{z}}<0$, thus, the wave front which is represented by the wave vector, is negatively refracted with respect to $\mathrm{x}$-component and also, undergoes backward propagation according to the negative $\mathrm{z}$-component. In analogy to the super lens, as proposed by Sir.Pendry, HMM can possibly allow for secondary sub-wavelength focusing in free space based negative refraction. When the EM exits the lens in the positive dielectric medium, it is negatively refracted with respect to the wave inside the lens, thus leading to secondary focusing in the near field of the HMM. Figure 1d demonstrates the travelling wave vector and Poynting vector of a single ray throughout the HMM. 

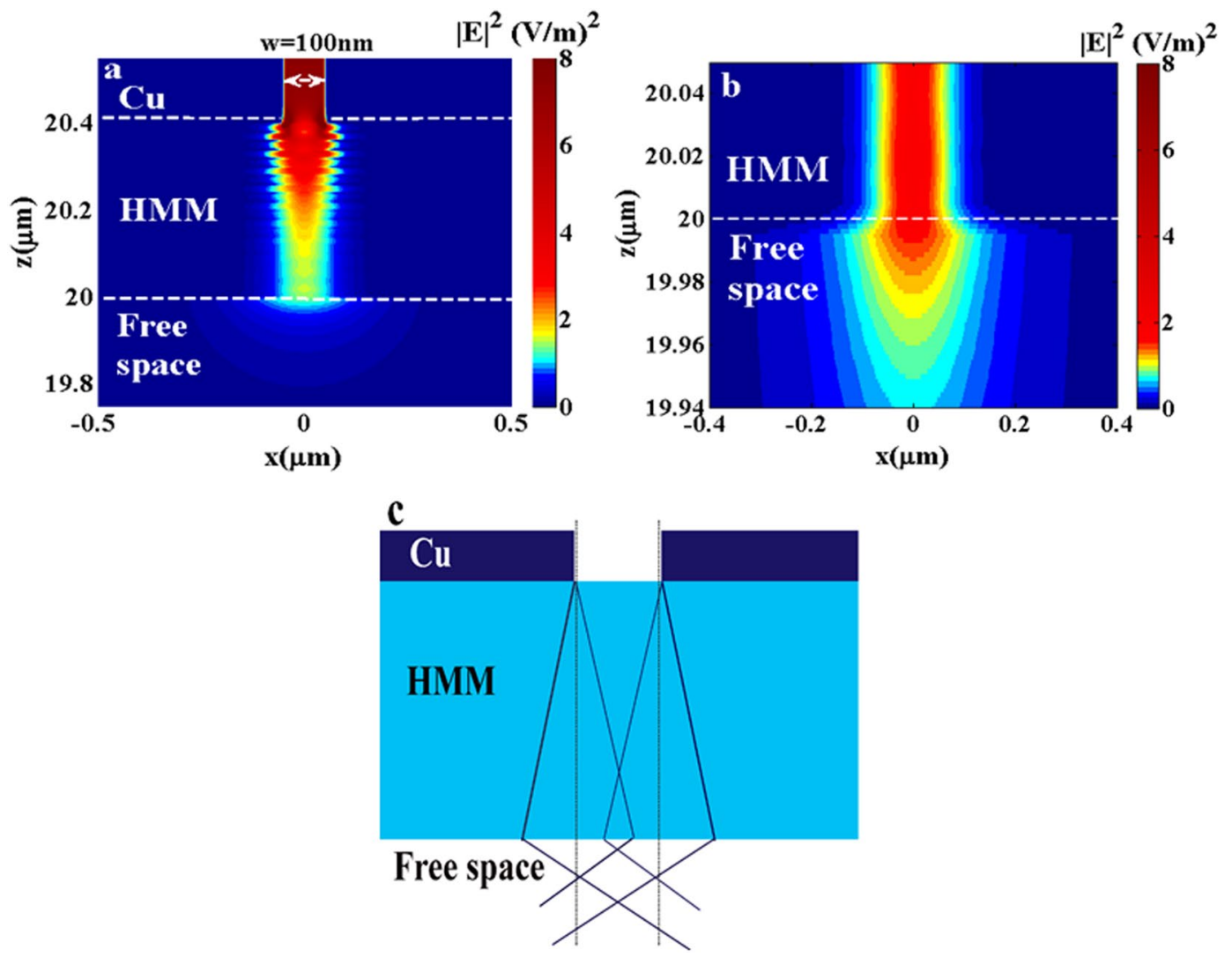

Figure 3. The electric field intensity distributions in xz-plane: (a) the whole structure is of slit width $100 \mathrm{~nm}$ and (b) zoom in across the HMM/free space interface, confirms that the propagating field undergoes negative refraction in the near field of the HMM lens and (c) The schematic is presenting the ray tracing diagram throughout the structure; the dashed lines represent the optical axes, the diagram shows the expected negative refraction in the near field of the HMM.

\section{Results and Discussion}

We verify the effect of focusing in the near field of HMM through a comparison of the electric field distribution throughout two structures: intrinsic InAs and HMM lens using effective medium approximation theory, Fig. 2. It is clearly observed that there is almost no propagation of the electric field in intrinsic InAs while, in HMM, the electric field travels throughout and transmits out of HMM in form of focused waves. Figure $2 \mathrm{c}$ confirms the focusing effect through the normalized electric field profile measured at a plane lies $500 \mathrm{~nm}$ away from the HMM.

In the next section, we apply the real structure and investigate the sub-diffraction focusing effect in the near field, Fig. 3. Figure 3(a,b) shows that the major part of the electric field which is excited at both edges of metallic slit, travels throughout the HMM with a small angle of refraction with focusing inside the lens and also extended outside the lens. Part of the electric field transmits outside the lens and gets focused in the near field. Figure $3 \mathrm{~b}$ represents zoom in across the $\mathrm{HMM}$ /free space interface confirming the negative refraction behavior outside the lens, and thus focusing in the near field of the lens. It is observed in the same figure the presence of broadening in the electric field in the very near field of the lens. This broadening is confined within very short distance, so called transition distance. The transition distance is the distance at which the EM behaviour is in between the coupled surface plasmon polariton in multilayered structure -which can sustain the propagation of the high wave vectors and results in bulk surface plasmon polariton (BSPP) -, and free space where the high $k$-modes start to exhibit different dielectric permittivity (positive medium). The BSPP provides enhanced localized density of states (LDOS) in the very near field of the structure ${ }^{28}$. As a result, the homogenization of the HMM within the transition distance breaks down and the propagating large wave vectors do contribute with deviation to the emerged large LDOS. After the transition distance, the HMM exhibits homogenization model and the propagating wave vectors start to propagate with negatively refraction behavior verifying a secondary focusing point in the near field. In ref. ${ }^{28}$, the transition distance is estimated to be around a/2pi -where a is the layer thickness- which is in good agreement with our results where the transition distance in the proposed structure is around $3 \mathrm{~nm}$, Fig. (3b).

Figure $3 \mathrm{c}$ shows a schematic for the ray tracing explaining the secondary negative refraction in the near field of HMM.

Now, we investigate the focusing resolution and the focusing resolution distance away from the HMM lens. We optimize the slit width $\boldsymbol{w}$ for obtaining the best focusing resolution at a plane lies $500 \mathrm{~nm}$ away from the HMM lens and also obtaining the maximum focusing distance that is possible away from the HMM lens. The focusing resolution is determined by the full width at half maximum (FWHM) of the electric field profile with respect to 

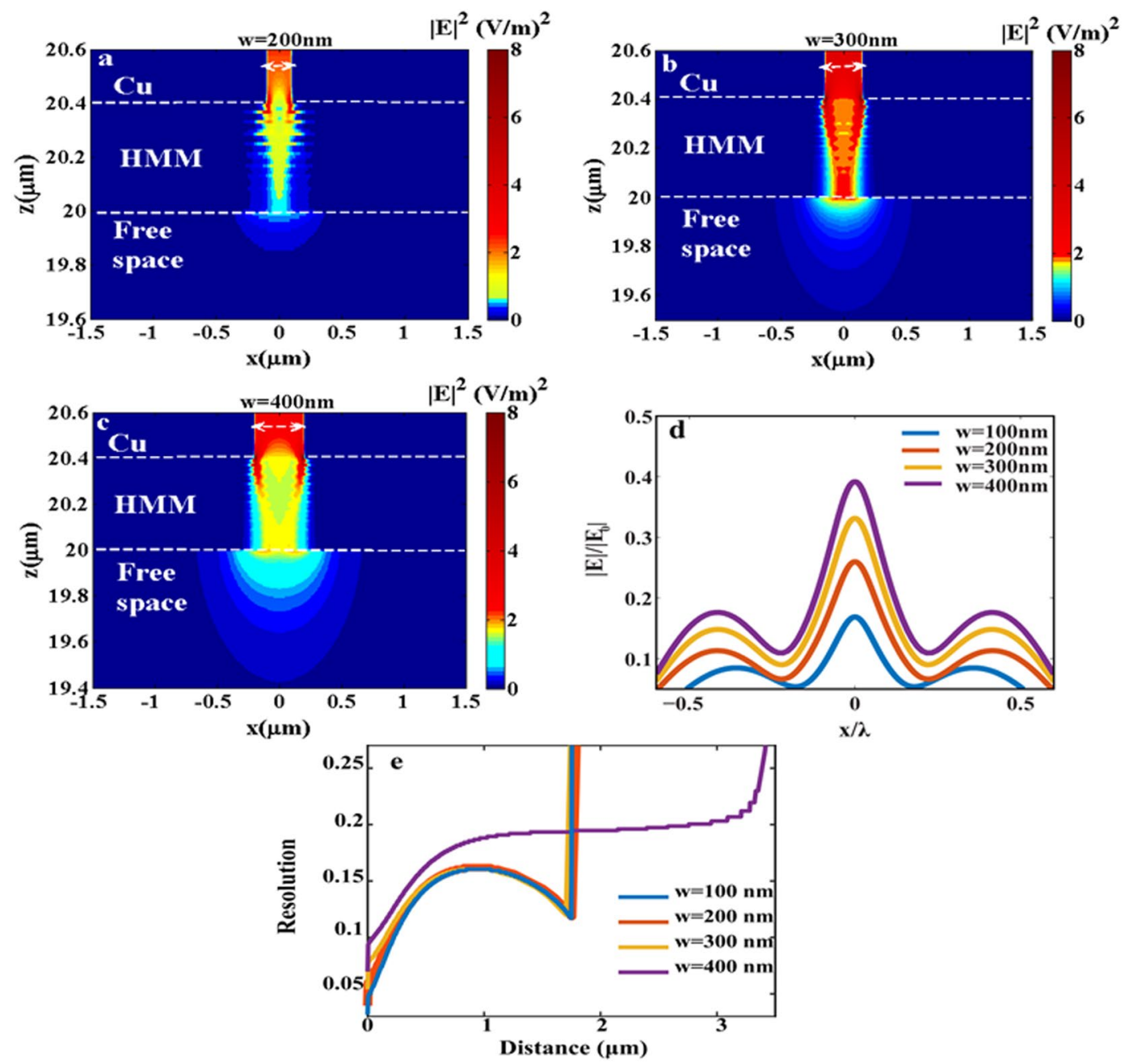

Figure 4. The electric field intensity distributions in xz-plane throughout a range of $w$ : (a) $200 \mathrm{~nm}$, (b) $300 \mathrm{~nm}$, (c) $400 \mathrm{~nm}$, demonstrating the focusing effect. (d) The normalized electric field profile in a plane at distance $500 \mathrm{~nm}$ below the HMM lens showing the direct relation between the $\boldsymbol{w}$ and amplitude of the normalized electric field while the focusing resolution is almost fixed around $(0.15 \lambda-0.16 \lambda)$ and (e) The focusing resolution against the distance away from the HMM lens showing the superior extended focusing of HMM of $\boldsymbol{w}$ of $400 \mathrm{~nm}$ to a distance of $3.2 \mu \mathrm{m}$, despite of its least resolution than other structures in short distances.

the incident wavelength $(\mathrm{x} / \lambda)$. The focusing effect is achieved within a range of $\boldsymbol{w}$ between $100 \mathrm{~nm}$ to $400 \mathrm{~nm}$, Fig. 4. Figure $4(\mathrm{a}-\mathrm{c})$ shows that the focusing resolution almost doesn't change with changing the $\boldsymbol{w}$ from 100 to $300 \mathrm{~nm}$ with focusing resolution down to $0.15 \lambda$, except for $\boldsymbol{w}$ of $400 \mathrm{~nm}$, where the resolution slightly changes to $0.16 \lambda$. However, the amplitude of the electric field is increased with increasing the slit width, Fig. $4 \mathrm{~d}$. Moreover, the structure of $w 400 \mathrm{~nm}$ exhibits extended focusing distance to $3.2 \mu \mathrm{m}$, Fig. 4e. Figure $4 \mathrm{e}$ also shows that the resolution for the structures of $w 100-300 \mathrm{~nm}$ decreases along the focal axis up to a point. After this point, the resolution starts to increase and then vanishes. This can be explained upon the demonstration of the scheme of the ray tracing, Fig. 3c. The scheme shows the arising of two separate focusing points. The first focusing point is arisen from the negative refraction of the focused rays inside the HMM. Meanwhile, the second focusing point is arisen from the negative refraction of the unfocused rays inside HMM. The region between the two focusing points exhibiting the least resolution as it is an extension of the two focusing points. For the structure of $w 400 \mathrm{~nm}$, it seems that the second focusing point is not well formed as the previous cases. Instead, the rays travel with negative refraction for long distance $(3.2 \mu \mathrm{m})$ exhibiting small resolution. Thus, there is a direct relation between the slit width and the focusing condition to be achieved. The slit width needs to be designed in away such that the first refracted rays with slight angles of propagation can interfere in a point inside the HMM in order to form the first focusing point outside the HMM. Also, the first refracted unfocused rays with slight angles of propagation inside the HMM can be narrow enough to project a second focusing point in the near field of the HMM. Then the refracted rays exiting the HMM undergoes secondary focusing, based on an opposing refraction to the rays inside the HMM. Thus, optimizing the $w$ is a crucial parameter for the near field focusing outside the HMM.

Finally, we examine focusing resolution with respect to the distance away from the HMM using different doping concentrations $\left(\mathrm{N}_{\mathrm{d}}\right)$ of $7.5 \times 10^{19} \mathrm{~cm}^{-3}, 4.4 \times 10^{19} \mathrm{~cm}^{-3}$ and $1 \times 10^{19} \mathrm{~cm}^{-3}$ corresponding to wavelengths of $7.6 \mu \mathrm{m}$, 


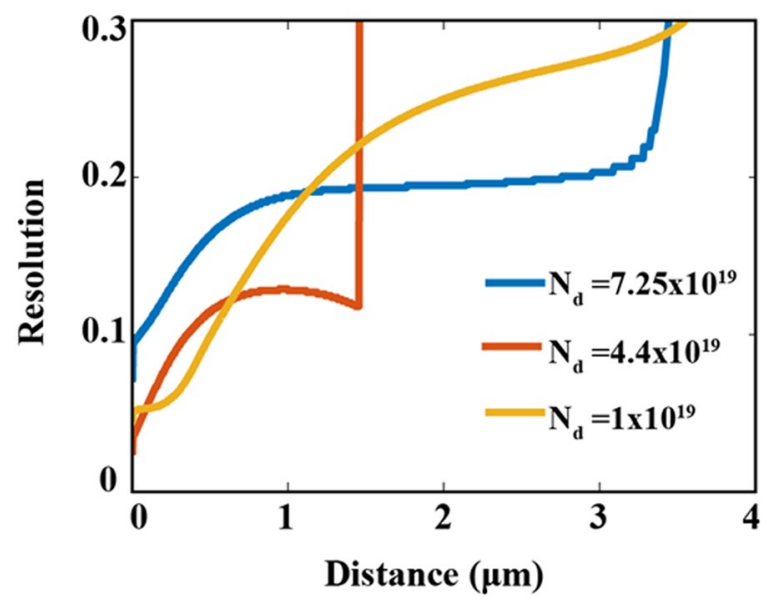

Figure 5. The focusing resolution against the distance away from HMM lens of slit width $w$ of $400 \mathrm{~nm}$ for different doping concentrations $\left(\mathrm{N}_{\mathrm{d}}\right)$ of $7.25 \times 10^{19}, 4.4 \times 10^{19}, 1 \times 10^{19} \mathrm{~cm}^{-3}$ corresponding to focusing wavelengths of $7.6 \mu \mathrm{m}, 9 \mu \mathrm{m}$ and $17.74 \mu \mathrm{m}$, respectively.

\begin{tabular}{|l|l|l|l|l|}
\hline & $\begin{array}{l}\text { Focusing } \\
\text { wavelength } \\
(\boldsymbol{\mu m})\end{array}$ & $\begin{array}{l}\text { Theoretical } \\
\text { inside angle of } \\
\text { refraction }(\boldsymbol{\theta})\end{array}$ & $\begin{array}{l}\text { Focusing } \\
\text { resolution at } \\
(\mathbf{5 0 0} \mathbf{~ n m})\end{array}$ & $\begin{array}{l}\text { Maximum } \\
\text { focusing } \\
\text { distance }(\boldsymbol{\mu m})\end{array}$ \\
\hline $7.25 \times 10^{19}$ & 7.6 & $10.9^{\circ}$ & $0.19 \lambda$ & 3.2 \\
\hline $4.4 \times 10^{19}$ & 9 & $3.79^{\circ}$ & $0.11 \lambda$ & 1.45 \\
\hline $1 \times 10^{19}$ & 17.74 & $7.03^{\circ}$ & $0.099 \lambda$ & 3.5 \\
\hline
\end{tabular}

Table 1. Summary of focusing effects according to the inside angle of propagation, the focusing resolution at a plane lies $500 \mathrm{~nm}$ away from the HMM surface, and the maximum focusing distance with respect to different doping concentration at their best focusing wavelengths.

$9 \mu \mathrm{m}$, and $17.74 \mu \mathrm{m}$ respectively, (these wavelengths have been selected based upon the best focusing resolutions achieved). Figure 5 and Table 1 show that the maximum sub-diffraction focusing distance achieved for each doping concentration is: $3.2 \mu \mathrm{m}, 1.45 \mu \mathrm{m}$ and $3.5 \mu \mathrm{m}$, respectively. At short distances up to $600 \mathrm{~nm}$, the region of the first elongated focusing point, the focusing resolution is greatly enhanced by decreasing the doping concentration. The reason behind is that by decreasing the doping concentration, the metallic property of the structure decreases and hence the imaginary part of the index of refraction that defines the losses decreases. However, at large distances beyond $600 \mathrm{~nm}$, the resolution is less affected by the metallic property and losses. The different doping concentrations exhibit different behaviour along the focal axis. In case of $\left(\mathrm{N}_{\mathrm{d}}\right)$ of $4.4 \times 10^{19} \mathrm{~cm}^{-3}$, the focusing resolution is enhanced at large distances due to the formation of the secondary focusing point as previously explained. In contrary, the case of $\left(\mathrm{N}_{\mathrm{d}}\right)$ of $7.5 \times 10^{19} \mathrm{~cm}^{-3}$ and $1 \times 10^{19} \mathrm{~cm}^{-3}$, the focusing resolution gradually decreases till vanishes. Thus, the control of energy losses emerged from either doping concentration or even by fabrication roughness is crucial for enhancing the focusing resolution in the near region of the near field of HMM.

Table 1 summarizes the values of the inside angle of refraction, the focusing resolution at a plane lies $500 \mathrm{~nm}$ away from the HMM, and the maximum extended focusing distance with respect to different doping concentration with their best focusing wavelengths indicated.

\section{Conclusion}

In this work, we theoretically demonstrate near field sub-diffraction focusing in free space using HMM lens based on InAs semiconductors in the mid-IR range. Focusing is achieved using HMM type (П) as they support paraxial propagation of high $k$-modes. Hence, negative refraction of energy is achievable across the interface of HMM $\backslash$ free space and consequently sub-diffraction focusing in the near field is realized. We optimized the structure parameters for achieving the highest resolution and maximum focusing distance away from the HMM lens with best achieved resolution down to $0.2 \lambda$ at maximum distance of $3.2 \mu \mathrm{m}$ for $\mathrm{N}_{\mathrm{d}}$ of $7.25 \times 10^{19} \mathrm{~cm}^{-3}$. Upon using different doping concentrations, we find that the small doping concentrations enhance the resolution in short distances from the HMM up to $600 \mathrm{~nm}$. The sub-diffraction resolution for $\mathrm{N}_{\mathrm{d}}$ of $7.25 \times 10^{19} \mathrm{~cm}^{-3}, 4.4 \times 10^{19} \mathrm{~cm}^{-3}, 1 \times 10^{19}$ $\mathrm{cm}^{-3}$ at wavelengths of $7.6 \mu \mathrm{m}, 9 \mu \mathrm{m}$ and $17.74 \mu \mathrm{m}$ are $0.16 \lambda, 0.11 \lambda$, and $0.099 \lambda$ respectively projecting maximum focusing distances up to $3.2 \mu \mathrm{m}, 1.45 \mu \mathrm{m}$ and $3.5 \mu \mathrm{m}$, respectively. Our study engenders new paradigms to realize mid-IR metalens for thermal imaging and photolithography application.

\section{Materials and Methods}

Finite difference time domain (lumerical software) has been used for simulating the optical response of the proposed structures to incident plane waves. Material modeling using Drude model has been performed using Matlab. 
Received: 15 July 2019; Accepted: 6 January 2020;

Published online: 13 July 2020

\section{References}

1. Jacob, Z., Alekseyev, L. V. \& Narimanov, E. Optical hyperlens: far-field imaging beyond the diffraction limit. Opt. Express. 14, 8247-8256 (2006).

2. Fischer, U. C. \& Zingsheim, H. P. Submicroscopic pattern replication with visible light. J. Vac. Sci. Technol. 19, 881-885 (1981).

3. Hecht, B. et al. Scanning near-field optical microscopy with aperture probes: Fundamentals and applications. J. Chem. Phys. 112, 7761-7774 (2000).

4. Veselago, V. G. The electrodynamics of substances with simultaneously negative values of $\varepsilon$ and $\mu$. Sov. Phys. Uspekhi. 10, 509-514 (1968).

5. Pendry, J. B. Negative refraction makes a perfect lens. Phys. Rev. Lett. 85, 3966-3969 (2000).

6. Hoffman, A. J. et al. Negative refraction in semiconductor metamaterials. Nat. Mater. 6, 946-950 (2007).

7. Response, T. M. et al. Terahertz magnetic response from artificial materials. Science. 303, 1494-1496 (2004).

8. Zhou, F. et al. Hiding a realistic object using a broadband terahertz invisibility cloak. Sci. Rep. 1, 78 (2011).

9. Tao, H. A metamaterial absorber for the terahertz regime: Design, fabrication and characterization. Opt. Express 16, 7181-7188 (2008).

10. Shao, L. et al. Dielectric 2-bit coding metasurface for electromagnetic wave manipulation. Journal of Applied Physics 125, 203101 (2019).

11. Shao, L. et al. Dual-functional coding metasurfaces made of anisotropic all-dielectric resonators. IEEE Access 7, 45716-45722 (2019).

12. Zhu, W. et al. Coherent perfect absorption in an all-dielectric metasurface. Applied Physics Letters 108, 121901 (2016).

13. Grady, N. K. et al. Terahertz metamaterials for linear polarization conversion and anomalous refraction. Science 340, 1304-1307 (2013).

14. Lee, H. et al. Realization of optical superlens imaging below the diffraction limit. New J. Phys. 7, 255 (2005)

15. Liu, Z., Fang, N., Yen, T. J. \& Zhang, X. Rapid growth of evanescent wave by a silver superlens. Appl. Phys. Lett. 83, 5184-5186 (2003).

16. Fang, N., Liu, Z., Yen, T.-J. \& Zhang, X. Regenerating evanescent waves from a silver superlens. Opt. Express. 11, 682 (2003).

17. Poddubny, A., Iorsh, I., Belov, P. \& Kivshar, Y. Hyperbolic metamaterials. Nat. Photonics. 7, 958-967 (2013).

18. Taubner, T. et al. Near-field microscopy through a SiC superlens. Science. 313(5793), 1595 (2006).

19. Smolyaninov, I. I. \& Smolyaninova, V. N. Hyperbolic metamaterials: Novel physics and applications. Solid. State. Electron. 136, 102-112 (2017).

20. Shekhar., P., Atkinson, J. \& Jacob, Z. Hyperbolic metamaterials: fundamentals and applications. Nano Convergence. 1, 1-17 (2014).

21. Liu, Z. et al. Far-field optical hyperlens magnifying sub-diffraction limited objects. Science. 315, 1686 (2012).

22. Guo, K., Liu, J., Zhang, Y. \& Liu, S. Chromatic aberration of light focusing in hyperbolic anisotropic metamaterial made of metallic slit array. Opt. Express 20, 28586 (2012).

23. Pan, R., Tang, Z., Pan, J. \& Peng, R. Tuning subwavelength-structured focus in the hyperbolic metamaterials. Proceedings of the SPIE, 10154, (2016)

24. Desouky, M., Mahmoud, A. M. \& Swillam, M. A. Tunable Mid IR focusing in InAs based semiconductor Hyperbolic Metamaterial. Sci. Rep. 7, 15312 (2017).

25. Ni, X. et al. Loss-compensated and active hyperbolic metamaterials. Opt. Express. 19, 25242 (2011).

26. Kaur, A. et al. Deep subwavelength power concentration- based hyperbolic metamaterials. Int. J. Opt. 2012, 879392 (2012).

27. Kannegulla, A. \& Cheng, L.-J. Subwavelength focusing of terahertz waves in silicon hyperbolic metamaterials. Opt. Lett. 41, 3539 (2016).

28. Peragut, F. et al. Hyperbolic metamaterials and surface plasmon polaritons. Optica. 4, 1409 (2017).

29. Soukoulis, C. M. \& Wegener, M. Optical metamaterials-more bulky and less lossy. Science 330, 1633-1634 (2010).

30. Valentine, J. et al. Three-dimensional optical metamaterials with negative refractive index. Nature 455, 376-379 (2008).

31. Zhang, X., Debnath, S. \& Güney, D. Ö. Hyperbolic metamaterial feasible for fabrication with direct laser writing processes. JOSA B. 32, 6 (2015).

32. Wei, D. et al. Single-material semiconductor hyperbolic metamaterials. Opt. Express. 24, 8735-8745 (2016).

33. High-performance Nanophotonic simulation software - Lumerical. available at: https://www.lumerical.com/. (Accessed: 29th November 2018).

34. Lalanne, P. \& Lemercier-Lalanne, D. On the effective medium theory of subwavelength periodic structures. J. Mod. Opt. 43, 2063-2086 (1996).

35. Law, S., Adams, D. C., Taylor, A. M. \& Wasserman, D. Mid-infrared designer metals. IEEE Photonics Conf. IPC. 20, 786-787 (2012).

36. Sayem, A. A., Mahdy, M. R. C. \& Rahman, M. S. Broad angle negative refraction in lossless all dielectric or semiconductor based asymmetric anisotropic metamaterial. J. Opt. 18, 1 (2015).

37. Shelby, A. R. A., Smith, D. R. \& Schultz, S. Experimenta verification of a negative index of refraction. Science. 292, 77-79 (2016)

38. Ferrari, L. et al. Hyperbolic metamaterials and their applications. Prog. Quantum Electron. 40, 1-40 (2015).

39. Foteinopoulou, S. \& Soukoulis, C. M. Negative refraction and left-handed behavior in two-dimensional photonic crystals. Phys. Rev. B 67, 235107 (2003).

40. Dumelow, T., Da Costa, J. A. P. \& Freire, V. N. Slab lenses from simple anisotropic media. Phys. Rev. B - Condens. Matter Mater. Phys. 72, 1-8 (2005).

41. Liu, Y., Bartal, G. \& Zhang, X. All-angle negative refraction and imaging in a bulk medium made of metallic nanowires in the visible region. Opt. Express. 16, 15439 (2008).

\section{Acknowledgements}

The authors would like to thank the American university in Cairo and the Academy of Scientific Research and Technology (ASRT) for financial support.

\section{Author contributions}

M.S. has suggested the concept and supervised the project. N.S. has performed the final design, theoretical modelling and numerical simulations. N.S. and M.D. have written the manuscript. S.O. has contributed to the technical discussion regarding metamaterial physical concepts and related design issues presented in the paper. All the author have revised and edited the manuscript.

\section{Competing interests}

The authors declare no competing interests. 


\section{Additional information}

Correspondence and requests for materials should be addressed to M.A.S.

Reprints and permissions information is available at www.nature.com/reprints.

Publisher's note Springer Nature remains neutral with regard to jurisdictional claims in published maps and institutional affiliations.

(c) (i) Open Access This article is licensed under a Creative Commons Attribution 4.0 International License, which permits use, sharing, adaptation, distribution and reproduction in any medium or format, as long as you give appropriate credit to the original author(s) and the source, provide a link to the Creative Commons license, and indicate if changes were made. The images or other third party material in this article are included in the article's Creative Commons license, unless indicated otherwise in a credit line to the material. If material is not included in the article's Creative Commons license and your intended use is not permitted by statutory regulation or exceeds the permitted use, you will need to obtain permission directly from the copyright holder. To view a copy of this license, visit http://creativecommons.org/licenses/by/4.0/.

(C) The Author(s) 2020 